\title{
View and present status of personnel involved in clinical trials : a survey of participants from the First Symposium of the Shikoku Collaborative Group for Promotion of Clinical Trials
}

\author{
Hiroaki Yanagawa', Minoru Irahara', Hitoshi Houchi², Yoshiyuki Kakehi², \\ Takashi Moritoyo ${ }^{3}$, Masahiro Nomoto ${ }^{3}$, Mitsuhiko Miyamura ${ }^{4}$, Taro Shuin ${ }^{4}$, and \\ the Shikoku Collaborative Group for Promotion of Clinical Trials \\ ${ }^{1}$ Clinical Trial Center for Developmental Therapeutics, Tokushima University Hospital ${ }^{2}$ Clinical Trials \\ Management Center, Kagawa University Hospital ; ${ }^{3}$ Clinical Pharmacology Center, Ehime University \\ Hospital ; and ${ }^{4}$ Clinical Trial Center, Kochi Medical School Hospital
}

\begin{abstract}
Clinical trials leading to drug approval (registration trials) play a central role in the drug development process. Since the introduction of the Good Clinical Practice (GCP) standard in 1997, the Japanese infrastructure for registration trials has improved. The contribution of support staff, including clinical research coordinators (CRCs), to clinical trials is now widely recognized in Japan. Quality issues and career development for these support staff are being increasingly emphasized. The Shikoku Collaborative Group for Promotion of Clinical Trials was organized in 2008 to address these issues through communication with the personnel involved in clinical trials in regional areas of Japan. To understand the views and present status of personnel involved in clinical trials, we used questionnaires to survey the participants of the First Symposium of the Shikoku Collaborative Group for Promotion of Clinical Trials held in August 2009. Group discussions and special lectures occurred at the symposium. The questionnaire began with questions about basic patient characteristics, followed by practical questions. Of 110 participants, there were 68 respondents $(62 \%)$, including clinical trial support staff (clinical research coordinators [n=36, 53\%], administrative officers $[n=9,13 \%]$, and medical staff $[n=23,34 \%])$. Among the support staff, $36(80 \%)$ had more than 5 years of experience. The most common questionnaire answer selected for participation in the symposium was "willing to contact staff from other medical institutions or organizations" for support staff and "to obtain further knowledge concerning clinical trials" for medical staff. The overall view of the discussion ("Was the discussion satisfactory?") was favorable for $36(53 \%)$ respondents. This survey revealed that the group discussion in the present symposium appears to be valuable for participants, using overall satisfaction as a surrogate. Based on the information obtained in the present study, further development of the clinical trial infrastructure, including training opportunities and career development for support staff, is required. Due to the limitations of this study, further analysis is warranted to determine the optimal strategy for training support staff. J. Med. Invest. 58 : 81-85, February, 2011
\end{abstract}

Keywords : clinical trial, clinical research coordinator, training, regional area

Received for publication November 12, 2010 ; accepted November $30,2010$.

Address correspondence and reprint requests to Hiroaki Yanagawa, M.D., Ph.D., Clinical Trial Center for Developmental Therapeutics, Tokushima University Hospital Kuramoto-cho 2, Tokushima 770-8503 Japan and Fax : +81-88-633-9295.

\section{INTRODUCTION}

Clinical trials leading to drug approval (registration trials) play a central role in the drug development process, and clinical trials in the general 
practice setting are important for providing evidence about the efficacy and safety of different agents in various settings. Since the introduction of the Good Clinical Practice (GCP) standard in 1997 and the plan for the promotion of registration trials by the Ministry of Health, Labor and Welfare and the Ministry of Culture and Science of Japan, the Japanese infrastructure for registration trials has improved. For example, the contribution of clinical research coordinators (CRCs) to clinical trials is now widely recognized. Recently, a marked increase both in the absolute numbers and in the percentage of clinical trial notifications of global clinical trials that include Japanese subjects has been observed (1), and conducting clinical trials has become increasingly complicated in Japan as well as in the global settings (2). Quality issues regarding support staff, in addition to investigators, are now being emphasized to ensure celerity and high quality in clinical trial conduct.

To address clinical trial support staff quality issues, various certification systems have been developed by societies, such as the CRC certification by the Japanese Society for Clinical Pharmacology and Therapeutics, and nationwide opportunities for learning and training have also been developed. In addition, person-to-person communication and discussion of issues, including support staff quality issues, appears to be a reasonable strategy for promoting clinical trials among personnel involved in clinical trials in regional areas. For this purpose, the Shikoku Collaborative Group for Clinical Trials was organized in 2008 by the personnel of departments concerned with promotion of clinical trials in the four university hospitals in the Shikoku area of Japan. Since understanding the view and present status of personnel involved in clinical trials may contribute to future activity of the group, we used questionnaires to survey the participants of the First Symposium of the Shikoku Collaborative Group for Clinical Trials held in August 2009 at Tokushima University Hospital. Herein we present the results of this analysis.

\section{METHODS}

The symposium was open to medical staff, contract research organization staff, and site management organization staff, and consisted of two parts. The first part involved group discussions about issues facing clinical trial management, such as basic activity of CRCs, promotion of clinical trial enrollment, multi-national clinical trials, CRC career paths, and the administrative work involved in clinical trials. The participants discussed each of these issues for approximately 1.5 hours in small groups $(n=8$ 12). The second part consisted of special lectures presented by clinical trial experts. After the symposium, a questionnaire was given to each participant. The questionnaire began with questions about basic participant characteristics. This was followed by practical questions about issues such as the main reason for participating in the symposium and views on present training opportunities using a five-point scale (strongly agree, agree, neutral, disagree, and strongly disagree).

We compared the views of clinical trial support staff (CRCs and administrative officers) with those of medical staff, and categorical variables were analyzed using the $\chi^{2}$ test. $P$ values $<0.05$ were considered to be statistically significant. All $P$ values were based on two-sided tests.

\section{RESULTS}

\section{Respondent characteristics}

Of 110 symposium participants, 68 questionnaire respondents $(62 \%)$ were included in this analysis. These respondents included CRCs $(n=36,53 \%)$, administrative officers $(n=9,13 \%)$, and medical staff (physicians, $n=3,4 \%$; pharmacists, $n=16,24 \%$; nurses, $n=3,4 \%$; and a clinical laboratory technician, $\mathrm{n}=1,2 \%$ ).

Among the clinical trial support staff (CRCs and administrative officers, $n=45), 36(80 \%)$ had more than 5 years of experience, whereas 9 (20\%) had less than 1 year of experience. With respect to previous experience with small-group clinical trial training, 17 respondents had never participated in smallgroup training (38\%), 9 had participated in smallgroup training one time (20\%), 9 had participated in it two times (20\%), and 3 had participated in it more than two times (7\%) ; 7 participants provided no answer.

\section{Certification of CRC respondents}

Only 7 (19\%) of the responding CRCs $(n=36)$ had already acquired certification by the Japanese Society for Clinical Pharmacology and Therapeutics, while 5 expressed their willingness for future acquisition of this certification. 


\section{Main reason for participating in the symposium}

Select answers to the survey questions are shown in Table 1. The main reasons for participation were as follows : willing to attend the first such symposium in the Shikoku district ( $n=17,25 \%)$, willing to contact staff from other medical institutions or organizations $(n=16,24 \%)$, to obtain further knowledge concerning clinical trials $(n=30,44 \%)$, and other $(n=5,7 \%)$. The "willing to contact staff from other medical institutions or organizations" answer was observed at a significantly higher frequency $(\mathrm{P}=0.008)$ in support staff $(\mathrm{n}=15,33 \%)$ compared to medical staff $(\mathrm{n}=1,4.3 \%)$, whereas the "to obtain further knowledge concerning clinical trials" response was observed at a significantly higher frequency $(\mathrm{P}=0.012)$ in medical staff $(\mathrm{n}=15,65 \%)$ compared to support staff $(n=15,33 \%)$.

\section{View of the training method itself}

As noted above, each participant discussed each issue for about 1.5 hours in small groups $(n=8-2)$. A considerable number of respondents (27 [40\%]) wanted to participate in discussions in groups consisting of fewer individuals $(<8)$, and $43(63 \%)$ wished to hold the discussions for a longer period of time. The discussions were open to medical staff, contract research organization staff, and site management organization staff. The majority of respondents $(44,65 \%)$ agreed that participation from sponsors, such as pharmaceutical companies, would be welcomed.

The overall views of the discussion ("Was the discussion satisfactory?") were as follows : strongly agree (12 [18\%]), agree (24 [35\%]), neutral (12 $[18 \%])$, disagree (3 [4\%]), and strongly disagree (1 [2\%]) ; 16 (24\%) respondents provided no answer.

\section{DISCUSSION}

Before the introduction of the CRC concept, investigators participating in Japanese registration trials performed virtually all tasks related to the trial, from patient care to administrative work, throughout the course of the study (3). The importance of the contribution of CRCs to clinical trials is now widely recognized, even in Japan. In our previous study conducted at the Tokushima University Hospital, over $80 \%$ of the doctors requested CRC support throughout the registration trial (4). In addition, we found that physicians who could recruit participants into a trial considered the presence of a support system with CRCs as the reason to participate in the trial (5).

In the global setting, Gets et al. (2) at the Tufts Center for the Study of Drug Development analyzed data on protocols and study conduct performance in clinical trials conducted between 1999 and 2005. These investigators reported that the number of unique procedures and the frequency of procedures per protocol had increased, and investigative site work burden to administer each protocol increased at an even faster rate. Additionally, study conduct performance-that is, cycle time and patient recruitment and retention rates-worsened. In Japan, a marked increase both in the absolute numbers and in the percentage of clinical trial notifications of global clinical trials that include Japanese subjects has occurred in recent years (1). While cancer and cardiovascular disease have been the major target diseases of these trials, the range has recently expanded to include other diseases.

In consideration of these circumstances, improving the skills of clinical trial support staff, such as CRCs and administrative staff, in addition to those

Table 1 Main reason for participating in the symposium

Total $(\mathrm{n}=68) \quad$ Support staff $(\mathrm{n}=45) \quad$ Medical staff $(\mathrm{n}=23)$

Willing to attend the first such symposium in the Shikoku district

$17(25.0 \%)$

$13(28.9 \%)$

$4(17.4 \%)$

Willing to contact staff from other medical institutions or organizations

$16(23.5 \%)$

$15(33.3 \%)^{*}$

$1(4.3 \%)$

To obtain further knowledge concerning clinical trials

$30(44.1 \%)$

$15(33.3 \%)$

$15(65.2 \%)^{* \star}$

Other

$5(7.4 \%)$

$2(4.4 \%)$

$3(13.0 \%)$

** Significantly different $(\mathrm{P}=0.012)$ than support staff 
of the investigators, is now being emphasized at each medical institution to ensure efficient progress and improved quality of clinical trials. Moreover, support staff-specific issues, such as CRC career development, are now also identified as being important. The Shikoku Collaborative Group for Clinical Trials was organized in 2008 to fulfill these demands in the Shikoku area in Japan, and we conducted the present analysis to promote activity of the group by understanding the views and present status of the personnel involved in clinical trials.

To improve the skills of CRCs in Japan, obtaining and maintaining certification by the Japanese Society for Clinical Pharmacology and Therapeutics would appear to be desirable. However, in the present survey, few CRCs had already acquired or expressed their willingness for future acquisition of this certification. The possibility that regional differences, such as a relatively smaller number of clinical trials conducted in regional areas compared to urban areas, could result in the "less" requirement for skilled CRCs should be considered. In the group discussions about CRC career development, many CRCs insisted that there is little incentive to becoming a CRC and that no organized career path for CRCs exists (data not shown). Development of a clear CRC career path is an important issue for this collaborative group to resolve.

Regarding the reason for participating in the present symposium, more supportive staff than medical staff showed their willingness to contact staff from other medical institutions or organizations. From the perspective of the CRC, medical staffs, such as ward-based clinical nurses work as a nursing team. On the other hand, CRCs must be able to competently perform their research roles and must adapt to working alone and working with a variety of clinical professionals. CRCs often feel insecure and feel that they are perceived as a minority group, and that their complaints cannot be accepted by their colleagues who lack understanding and insight into the research process (6). Feelings of isolation and tension throughout clinical trials exist, even after CRCs have gained skills and confidence in their roles (7). Although it is now widely accepted that CRCs play important roles in ensuring the quality of clinical trials while lessening the workload of physicians, networking of CRCs, such as in the present collaborative group, could be beneficial for the support staff, including CRCs, to lessen their feelings of isolation, and may contribute to more efficient job performance.
Various methods exist for training medical staff, including CRCs. Compared to self-study, investigator meetings, and lectures, small-group discussions have unique value in their interactivity and immediate feedback. In the present analysis, discussions of approximately 1.5 hours in length in small groups $(n=8-12)$ appeared to be unsatisfactory to the participants. Longer discussion periods with participation of various professionals, including monitors and audit-related individuals from sponsors, such as pharmaceutical companies, should be considered for future small-group discussions. Taekman et al . (8) published a preliminary report on the use of high-fidelity simulation in the training of study coordinators conducting a clinical research protocol. Improvement of training methodology and evaluation of the contribution of training to clinical trial quality should be considered as a future goal of this collaborative group.

The present study evaluated only a small number of professionals involved in clinical trials. Nevertheless, the group discussion format used in the present symposium appears to be of value to participants when overall satisfaction is used as a surrogate. It is necessary to develop the proper infrastructure, including training opportunities, in the Shikoku area, based on the information obtained in the present study. Because of the study limitations, further study is warranted to determine the generalizability of the present findings to other Japanese regional areas.

\section{ACKNOWLEDGEMENTS}

The authors would like to thank Soichiro Tajima of the Clinical Trial Center for Developmental Therapeutics, Tokushima University Hospital for his help in preparing the manuscript.

\section{REFERENCES}

1. Ichimaru K, Toyoshima S, Uyama Y : Effective global drug development strategy for obtaining regulatory approval in Japan in the context of ethnicity-related drug response factors. Clin Pharmacol Ther 87 : 362-366, 2010

2. Getz KA, Wenger J, Campo RA, Seguine ES, Kaitin KI : Assessing the impact of protocol design changes on clinical trial performance. Am J Ther 15 : 450-457, 2008 
3. Ebihara A, Takahashi K, Ikemoto F, Yamamoto $\mathrm{K}$ : Clinical pharmacology and clinical trials in Japan. J Mol Med 74 : 479-486, 1996

4. Yanagawa $H$, Nishiya $M$, Miyamoto $T$, Shikishima M, Imura M, Nakanishi R, Ariuchi N, Akaishi A, Takai S, Abe S, Kisyuku M, Kageyama C, Sato C, Yamagami M, Urakawa $\mathrm{N}$, Sone S, Irahara M : Clinical trials for drug approval : a pilot study of the view of doctors at Tokushima University Hospital. J Med Invest 53 : 292-296, 2006

5. Yanagawa H, Kishuku M, Akaike M, Azuma $\mathrm{H}$, Irahara $\mathrm{M}$ : View of physicians on and barriers to patient enrollment in a multicenter clinical trial : experience in a Japanese rural area.
Int Arch Med $3: 7,2010$

6. Raja-Jones $\mathrm{H}$ : Role boundaries-research nurse or clinical nurse specialist? A literature review. J Clin Nurs 11 : 415-420, 2002

7. Spilsbury K, Petherick E, Cullum N, Nelson A, Nixon J, Mason S: The role and potential contribution of clinical research nurses to clinical trials. Crit Care Nurs $17: 549-557,2007$

8. Taekman JM, Hobbs G, Barber L, PhillipsBute BG, Wright MC, Newman MF, StaffordSmith M : Preliminary report on the use of high-fidelity simulation in the training of study coordinators conducting a clinical research protocol. Anesth Analg 99 : 521-527, 2004 